\title{
Conductancia hidráulica foliar y vulnerabilidad a la cavitación disminuyen con la altitud en Phacelia secunda J.F. Gmel. (Boraginaceae)
}

\author{
Hydraulic leaf conductance and cavitation vulnerability decrease with elevation in \\ Phacelia secunda J.F. Gmel. (Boraginaceae)
}

\author{
Carolina Hernández-Fuentes ${ }^{1,2} 2^{*}$, León A. Bravo ${ }^{3}$ \& Lohengrin A. Cavieres ${ }^{1,2}$ \\ 1Departamento de Botánica, Facultad de Ciencias Naturales y Oceanográficas, Universidad de Concepción. Casilla \\ 160-C, Concepción, Chile. \\ ${ }^{2}$ Instituto de Ecología y Biodiversidad (IEB), Las Palmaras 3425, Ñuñoa, Santiago, Chile. \\ ${ }^{3}$ Laboratorio de Fisiología y Biología Molecular Vegetal, Instituto de Agroindustria, Departamento de Ciencias \\ Agronómicas y Recursos Naturales, Facultad de Ciencias Agropecuarias y Forestales \& Center of Plant, Soil \\ Interaction and Natural Resources Biotechnology, Scientific and Technological Bioresource Nucleus. Universidad \\ de La Frontera, Casilla 54D, Temuco, Chile. \\ *carohernandez@udec.cl
}

\section{RESUMEN}

Los ecosistemas de alta montaña se caracterizan por presentar temperaturas bajo cero durante gran parte del año. Estos episodios de temperaturas bajo cero podrían inducir embolismo en las plantas que habitan estos ecosistemas ya que durante el congelamiento se generan burbujas de aire, las que pueden expandirse durante el descongelamiento conduciendo a la cavitación. La probabilidad de embolismo o cavitación en un conducto xilemático es inversamente proporcional a su diámetro. Sin embargo, la reducción del diámetro de los vasos, por pequeña que sea, disminuye considerablemente la conductividad de éstos. La conductancia hidráulica de la hoja $(K f)$ está definida como la proporción entre el flujo de agua a través de la hoja y la diferencia de potencial hídrico a través de esta. $K f$ es un cuello de botella para la conductancia hidráulica de la planta completa y probablemente es también uno de los factores más determinantes en el intercambio de gases. Dado que la intensidad y frecuencia de temperaturas congelantes cambia con la altitud, es importante estudiar cómo influye la altitud de procedencia de las plantas en la estructura e hidráulica de la hoja y las consecuencias de esto en las probabilidades de embolismo. En este trabajo aprovechamos la amplia distribución altitudinal de la hierba perenne Phacelia secunda para evaluar cómo cambia la conductividad hidráulica de la hoja y la vulnerabilidad a la cavitación con la altitud. Postulamos que en plantas de $P$. secunda provenientes de mayores altitudes, al estar expuestas a una mayor frecuencia de eventos congelantes, tienen un menor diámetro de vasos y con ello una disminución en la $K f$ comparado con plantas provenientes de menor altitud. Para evaluar esta hipótesis se determinó la conductancia hidráulica foliar $(K f)$ en plantas provenientes de diferentes altitudes $(1.600,2.800$ y $3.600 \mathrm{~m}$ ) en los Andes de Chile Central. Además, se realizaron cortes transversales de pecíolo y lámina para evaluar las variaciones en el número, tamaño y frecuencia de los conductos xilemáticos, y se determinó el índice de vulnerabilidad a la cavitación. Se encontró una correlación negativa entre la conductancia hidráulica de la hoja y la altitud. Lo anterior se relaciona con los menores diámetros de vasos xilemáticos encontrados en las plantas de mayor altitud, lo que trae como consecuencia una menor vulnerabilidad a la cavitación en dichas plantas. Lo anterior sugiere una ventaja adaptativa para estas plantas ya que a mayor elevación existe una mayor frecuencia de heladas y en consecuencia mayores probabilidades de cavitación. Sin embargo, esto podría tener un efecto negativo en las tasas de conductancia estomática y asimilación de carbono, disminuyendo el desempeño de estas plantas.

Palabras clave: Anatomía del xilema, embolismo, alta montaña, heladas.

\section{ABSTRACT}

High mountain ecosystems are characterized by freezing temperatures. Freeze-thaw events can induce embolism in plant xylem. The risk of embolism increases with the conduit diameter, because wider xylem vessels can contain larger amounts of dissolved gas that after water freeze may form big bubbles producing cavitation. Thus smaller vessels could be advantageous in habitats prone to freezing events. However, smaller conduits decrease hydraulic conductance. The leaf hydraulic conductance $(K f)$ is a measure of the efficiency in the water transport across the leaf, and it is defined as the ratio between the water flux across the leaf and the difference of hydraulic potential across the leaf. Apparently, $K f$ is a bottleneck for hydraulic conductance of the whole plant and probably also a crucial factor in gas exchange. In this work we evaluated how the elevational origin of $P$. secunda can affect the hydraulic conductance of the leaf and the cavitation vulnerability of the vessels. We postulate that plants of $P$. secunda from higher elevations, as exposed to higher frequency of freezing events, have minor vessels diameter and lower $K f$ than plants from lower elevations. To test our hypothesis, we determined under field conditions the leaf hydraulic conductance $(K f)$, in plants from an altitudinal gradient $(1,600,2,800$ and 3,600 $\mathrm{m})$ in the 
central Chilean Andes. In addition, we analyzed cross sections of petiole and leaf lamina to evaluate the changes in the number, size and frequency of xilematic vessels in order to determine a cavitation vulnerability index. We found a negative correlation between the hydraulic conductance of the leaf and elevation in P. secunda plants. This is related to the smaller diameter of xylem vessels found in plants higher altitude, which results in a reduced vulnerability to cavitation. These changes could be considered as adaptives, because at high elevations freezing events are more frequent, and hence, there is a higher probability of cavitation than at low elevations. However, this can negatively effect stomatal conductance and carbon assimilation, restricting the photosynthetic responses of these plants.

KEYworDs: Xylem anatomy, embolism, high mountain, freezing events.

\section{INTRODUCCIÓN}

El agua no sólo es responsable del turgor celular, fundamental para el crecimiento celular, sino que además proporciona apoyo en el sostén mecánico a plantas no leñosas y entre otros procesos es una fuente de electrones y protones para el proceso de fotosíntesis (Boyer 1976). Estudiar cómo es el flujo del agua y la arquitectura hidráulica de la planta completa es importante, ya que dichas características tienen consecuencias trascendentales en el crecimiento, funcionamiento, estructura de la hoja, y ecología de las plantas (Sack \& Tyree 2005).

La conductancia hidráulica de la hoja $(K f)$ es una medida de cuán eficiente es el transporte de agua a través de ésta (Mencuccini 2003, Tyree 2003, Poorter et al. 2010, Russo et al. 2010), y se define como la proporción entre el flujo de agua a través de la hoja (a través del pecíolo, venas y de los sitios de evaporación de la hoja) y la diferencia de potencial hídrico necesaria para obtener dicho flujo (Sack \& Holbrook 2006). La $K f$ es el inverso de la resistencia hidráulica de la hoja, y es típicamente normalizada por el área foliar, basado en el supuesto de que el número de vías paralelas para el flujo del agua podría incrementar linealmente con el área de la hoja (Martre et al. 2001, Sack et al. 2004). Existe evidencia que la $K f$ es un cuello de botella para la conductancia hidráulica de la planta completa y probablemente es también uno de los factores determinantes en el intercambio de gases (Hubbard et al. 2001, Brodribb et al. 2007). Lo anterior se debería a que el potencial hídrico de la hoja y el control de los estomas se encuentra fuertemente determinado por la $K f$, influyendo en el balance hídrico de la planta completa (Nardini \& Salleo 2000, Tyree \& Zimmermann 2002, Sack et al. 2003).

Las bajas temperaturas disminuyen la solubilidad de los gases en el agua provocando la formación de burbujas en el sistema vascular de las plantas. Esto es particularmente evidente en ambientes con una alta frecuencia de heladas. Los eventos de congelamiento-descongelamiento inducen embolismo debido a que el aire no es soluble en el hielo, generándose burbujas durante el congelamiento, las que pueden expandirse durante el descongelamiento, conduciendo a la cavitación (Mayr \& Cochard 2003). Los embolismos disminuyen el flujo de agua a través de los conductos xilemáticos (Boehm 1893, Dixon \& Joly 1894, Richter 1972, Jackson \& Grace 1994), llegando a ser letales en algunos casos (Mayr et al. 2014).

La probabilidad de embolismo en un conducto xilemático es inversamente proporcional a su diámetro (Choat et al. 2011). Es por esto que en plantas que crecen a bajas temperaturas, usualmente los vasos xilemáticos son más estrechos que en plantas que habitan ambientes más cálidos (Hacke \& Sperry 2001). Mientras la reducción del diámetro de los vasos reduce la probabilidad de embolismo inducido por frío, dicha reducción, por pequeña que sea, disminuye considerablemente la conductividad de los vasos (Davis et al. 1999, Jiménez-Castillo \& Lusk 2013). De acuerdo a la ley de Hagen-Poiseuille, la conductancia hidráulica de un vaso es directamente proporcional a la cuarta potencia de su diámetro (Zimmermann 1983, Ewers \& Fischer 1989). Es conocido que hojas con vasos de diámetros pequeños tienen una baja conductancia hidráulica, y correspondientemente una baja conductancia estomática (Sack \& Frole 2006), afectando el intercambio gaseoso de estas plantas (Hubbard et al. 2001). Sin embargo, se ha observado que en algunas ocasiones la modificación en el diámetro de los vasos va acompañada con una modificación en el número de ellos (McCulloh et al. 2003, 2004, Price et al. 2007, Atala \& Lusk 2008).

A lo largo de gradientes altitudinales en zonas de altamontaña, la temperatura ambiental disminuye con la altitud (Körner 2003). Lo anterior sugiere que en especies que presentan una amplia distribución altitudinal, sus poblaciones extremas debiesen mostrar estrategias diferentes para enfrentar las contrastantes condiciones ambientales. Por ejemplo, la tolerancia a temperaturas congelantes es mayor en poblaciones de Hordeum comosum J. Presl, Perezia carthamoides (D. Don) Hook. et Arn. y Taraxacum officinale G. Weber ex F.H. Wigg. ubicadas en altitudes mayores (Sierra-Almeida et al. 2009). En los Andes de Chile central, varias especies presentan una amplia distribución altitudinal, siendo una de éstas Phacelia secunda J.F. Gmel. (Boraginaceae), una hierba perenne que se distribuye desde los 1.600 a los 3.600 m s.n.m. (Cavieres 2000). El amplio rango altitudinal presentado por esta especie la hace un buen modelo de estudio, en relación a los cambios de su sistema conductor frente a los riesgos de embolismo por frío. Antecedentes previos han mostrado que $P$. secunda presenta una marcada variación altitudinal en su morfología, desde plantas con rosetas pequeñas, acaulescentes, de hojas erectas, con uno o dos tallos florales altos a baja altitud hasta plantas con rosetas grandes, con hojas y tallos florales cortos que crecen a ras de suelo (Cavieres 2000), sugiriéndose diferenciación ecotípica entre poblaciones ubicadas a distintas elevaciones (Cavieres \& Arroyo 1999). Mientras en las poblaciones de esta especie 
ubicadas a $1.600 \mathrm{~m}$ la temperatura promedio anual es $12,7^{\circ} \mathrm{C}$, en las poblaciones ubicadas a $3.200 \mathrm{~m}$ dicha condiciones es de $3{ }^{\circ} \mathrm{C}$ (Cavieres \& Arroyo 1999). Registros recientes de las condiciones de temperatura durante la estación de crecimiento indican que mientras a $2.700 \mathrm{~m}$ sólo el $9 \%$ de los días muestran eventos de temperatura congelante $\left(<0{ }^{\circ} \mathrm{C}\right)$, a $3.500 \mathrm{~m}$ el $45 \%$ de los días muestra tal condición (Cavieres et al. 2007). Por lo tanto, en plantas de $P$. secunda provenientes de mayores altitudes, al estar expuestas a una mayor frecuencia de eventos congelantes, se espera que muestren una disminución en el diámetro de sus vasos, lo que determinaría una menor $K f$, con la consiguiente disminución del riesgo de cavitación por congelamiento.

En el presente estudio evaluamos cómo varía altitudinalmente la conductividad hidráulica de la hoja y la vulnerabilidad a la cavitación en plantas de $P$. secunda, una especie de amplia distribución altitudinal en los Andes de Chile central.

\section{MATERIALES Y MÉTODOS}

ESPECIE ESTUDIADA

Phacelia secunda es una hierba perenne presente en Argentina, Bolivia, Chile y Perú, y que en Chile presenta una amplia distribución tanto latitudinal como altitudinal. $\mathrm{Su}$ distribución abarca desde Parinacota $\left(18^{\circ} \mathrm{S}\right)$ hasta Tierra del Fuego $\left(54^{\circ} \mathrm{S}\right)$ y desde el nivel del mar hasta cerca del límite altitudinal de la vegetación andina (3.600 m) (Cavieres 2000).

RECOLECCIÓN DE MATERIAL DE ESTUDIO Y CARACTERIZACIÓN MICROCLIMÁTICA

El estudio se realizó con 30 plantas de Phacelia secunda, recolectadas al inicio de la estación de crecimiento a 1.600 , 2.800 y $3.600 \mathrm{~m}$ de altitud. Los sitios de muestreo se encuentran en la zona de alta montaña de la Cordillera de los Andes de Chile central $\left(33^{\circ} \mathrm{S}\right)$, distante $50 \mathrm{~km}$ de la ciudad de Santiago.

Se escogieron tres sitios de estudio, los cuales presentan variaciones en sus características mesoclimáticas. El sitio a menor altitud está localizado a los $1.600 \mathrm{~m}$, en la reserva de la naturaleza Yerba Loca $\left(33^{\circ} 10^{\prime} \mathrm{S}, 70^{\circ} 13^{\prime} \mathrm{W}\right)$. En esta altitud la estación de crecimiento usualmente comienza a mediados de septiembre y se extiende hasta fines de abril. Las precipitaciones anuales para este sitio son de $445 \mathrm{~mm}$, la cual cae predominantemente en forma de lluvia en el invierno y con eventos poco frecuentes en el verano (Santibáñez \& Uribe 1990), con variaciones en la temperatura media de $23{ }^{\circ} \mathrm{C}$ como máximas en verano y de $1{ }^{\circ} \mathrm{C}$ como mínimas en el invierno (Santibáñez \& Uribe 1990). El segundo sitio se encuentra ubicado a $2.800 \mathrm{~m}$ sobre el nivel del mar, en una ladera de exposición norte, cercana al centro de Ski La Parva $\left(33^{\circ} 21^{\prime} \mathrm{S}, 70^{\circ} 19^{\prime} \mathrm{W}\right)$. En esta altitud la estación de crecimiento comienza a mediados de octubre y se extiende hasta finales de abril, con una temperatura media del aire para la estación de $10,9^{\circ} \mathrm{C}$. El sitio de mayor elevación se encuentra a $3.600 \mathrm{~m}$ de altitud, en una ladera de exposición noreste $\left(33^{\circ} 19^{\prime} \mathrm{S}, 70^{\circ} 15^{\prime} \mathrm{W}\right)$. A esta elevación la estación de crecimiento comienza en diciembre y finaliza en marzo y la temperatura media del aire durante la estación de crecimiento es de $6,8^{\circ} \mathrm{C}$ (Cavieres et al. 2007). Las plantas ubicadas a 1.600 y $2.800 \mathrm{~m}$ fueron recolectadas en el mes de noviembre de 2009 y para $3.600 \mathrm{~m}$ en el mes de diciembre de 2009. Para cada altitud se registraron datos de temperatura con estaciones microclimáticas HOBO (Micro Station Data Logger H21002) ubicadas en cada uno de los sitios de estudio.

\section{Muestreo}

En cada altitud se seleccionaron 10 individuos de $P$. secunda de tamaño similar, los cuales fueron extraídos con sustrato, cuidando de no dañar la raíz. Estas plantas fueron llevadas al laboratorio, en donde a cada uno de los individuos seleccionados se les midió la conductancia hidráulica foliar y el contenido relativo de agua, como se describe más adelante. Posteriormente, se tomaron muestras foliares de estas plantas, las cuales se fijaron en FAA (10 ml de formalina: $5 \mathrm{ml}$ de ácido acético glacial: $50 \mathrm{ml}$ de etanol) para realizar posteriormente los análisis histológicos.

\section{CONDUCTANCIA HIDRÁUlicA FOLIAR $(K f)$}

La conductancia hidráulica foliar fue medida in vitro aplicando presiones conocidas a hojas escindidas y midiendo el flujo de agua a través de la hoja (Nardini et al. 2001). Se tomaron 5 plantas en cada una de las tres altitudes. Los pecíolos se cortaron bajo agua para evitar el ingreso de aire al xilema. Las hojas se mantuvieron sumergidas en agua y expuestas a la luz durante $1 \mathrm{~h}$. Luego el pecíolo de cada hoja se conectó a una manguera de diámetro pequeño, la cual estaba por el otro extremo sumergida en un vaso de precipitado que contenía agua en su interior. La hoja, conectada a la manguera, se puso dentro de un matraz de Erlenmeyer, donde se aplicaron con una bomba de vacío cuatro tensiones $(0,-23,-40$ y -80 MPa). Las hojas fueron puestas en agua dentro del matraz, para asegurarse de que no hubiera pérdida de agua por evaporación durante las mediciones. Además, para no tener problemas con el cierre estomático (Sack et al. 2002), las hojas fueron mantenidas a la luz antes y durante el experimento.

Luego, con los datos obtenidos se realizó una regresión lineal entre el flujo de agua que pasó a través de la hoja (medida como diferencias en peso del vaso precipitado) por unidad de tiempo $\left(\mathrm{s}^{-1}\right)$ y las 4 presiones aplicadas, en donde la pendiente de esta regresión es una medida de la conductividad hidráulica de la hoja $(K f)$. Además, posteriormente se midió el área foliar de las hojas utilizando una cámara digital y analizando las fotos con el programa SigmaScan Pro 5.0. Así $K f$ fue expresada en mmol agua $\mathrm{m}-2 \mathrm{~s}^{-1} \mathrm{MPa}^{-1}$.

CARACTERIZACIÓN histolóGICA DE VASOS XILEMÁTICOS

Hojas escindidas de cinco plantas provenientes de las tres altitudes en estudio se seccionaron en trozos de $5 \mathrm{~mm}$ de 
longitud, mediante un corte transversal al eje del pecíolo y de la lámina. Luego se fijaron en solución FAA, dejando actuar 5 días para lograr una correcta fijación. Después de la fijación, se realizaron cortes transversales de pecíolo y lámina a mano alzada, los cuales fueron observados con un microscopio dotado de una cámara, todas las fotografías fueron tomadas con un objetivo de 40x, para poder observar el tejido vascular de las plantas.

DiÁMETRO, NÚMERO Y FRECUENCIA DE VASOS XILEMÁTICOS El diámetro de los vasos xilemáticos de pecíolo y de la lámina (a nivel de la vena central) se calculó midiendo el diámetro de 20 vasos seleccionados al azar de las fotografías obtenidas de los cortes transversales realizados. Además, se determinó el número y la frecuencia de los vasos del xilema (vasos $\mathrm{mm}^{-2} \mathrm{de}$ área de xilema). La frecuencia de los conductos se estableció mediante las fotografías tomadas de los cortes transversales de lámina y pecíolo, contando los vasos que caían en un área de $25 \mathrm{~mm}^{2}$ y se contabilizó el número de conductos.

ÍNDICE DE VULNERABILIDAD A LA CAVITACIÓN

La susceptibilidad a daños durante la conducción de agua fue evaluada a través del Índice de vulnerabilidad a la cavitación, propuesto por Reyes-Santamaría et al. (2002), éste es:

$$
\mathrm{IV}=\mathrm{DV} / \mathrm{FV}
$$

Donde "DV" es el diámetro de los vasos y "FV" la frecuencia de éstos.

\section{ANÁLISIS ESTADísTICOS}

Los datos se analizaron utilizando un análisis de varianza (ANOVA), comprobando previamente la homogeneidad de varianza y normalidad de los datos. Para el caso del CRA y $K f$, se utilizó una ANOVA de una vía, en donde el factor fue la altitud. En cambio, para analizar las diferencias en el número y diámetro de los vasos y el índice de vulnerabilidad se realizó un ANOVA de dos vías, donde los factores fueron la altitud y tejido de la hoja (lámina/pecíolo). En ambos casos se realizó posteriormente una prueba Fisher para identificar los valores que representan diferencias significativas $(\mathrm{p}<0,05)$.

\section{RESULTADOS}

VARIABLES MESOCLIMÁTICAS

Las temperaturas mínimas diarias del aire, medidas durante la estación de crecimiento de las plantas para las tres elevaciones, variaron con la altitud (Fig. 1). Las temperaturas mínimas del aire para plantas de $1.600 \mathrm{~m}$ fueron en promedio de 11,97 ${ }^{\circ} \mathrm{C}$ llegando a los $2,9^{\circ} \mathrm{C}$ en el día más frío. A los $2.800 \mathrm{~m}$ se observó una disminución de la temperatura mínima, en donde el promedio fue de $7,5^{\circ} \mathrm{C}$ siendo la más baja de $-1,7^{\circ} \mathrm{C}$. Para los $3.600 \mathrm{~m}$ hubo una disminución mayor en las temperaturas alcanzando un promedio de solo $5,9^{\circ} \mathrm{C}$ y la temperatura más baja alcanzó los $-4,8^{\circ} \mathrm{C}$. Además, se encontraron diferencias entre la frecuencia de eventos de temperatura inferior a $0{ }^{\circ} \mathrm{C}$ en la estación de crecimiento. En 3.600 m se encontraron 14 eventos de temperaturas congelantes, en cambio en $2.800 \mathrm{~m}$

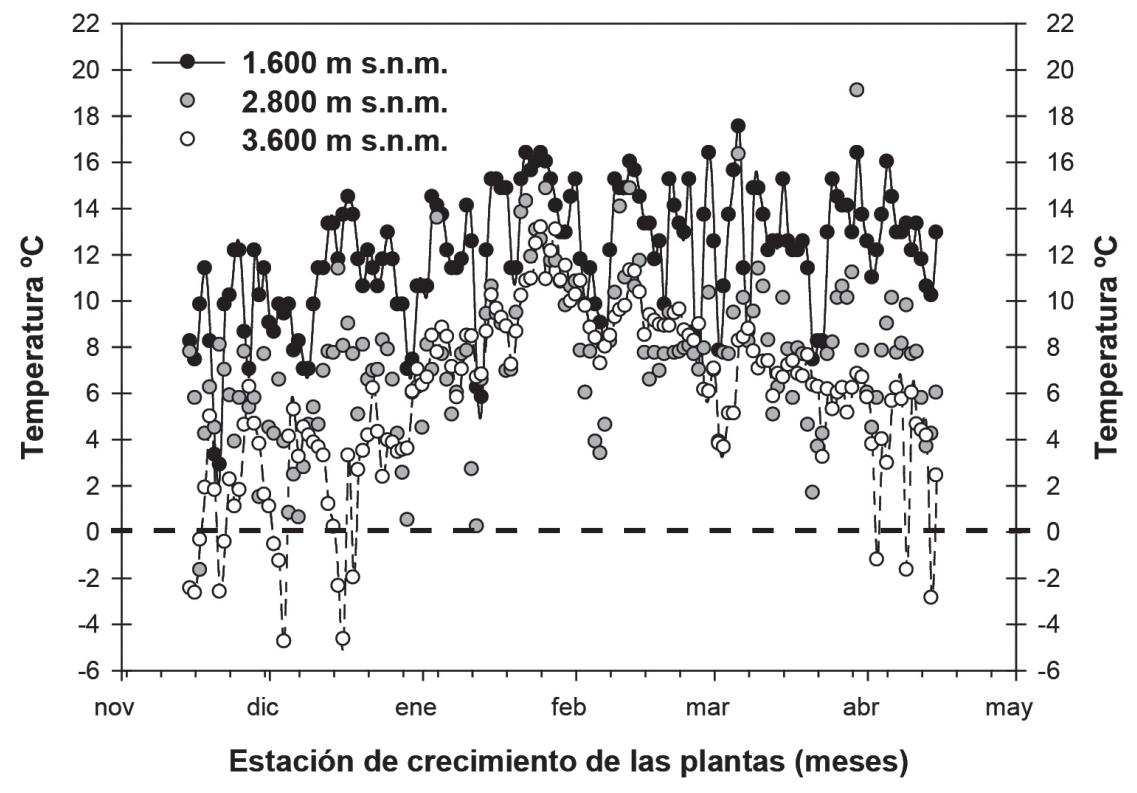

FiguRA 1. Temperaturas mínimas del aire registradas a los 1.600, 2.800 y 3.600 m s.n.m. en tres sitios de la Cordillera de los Andes de Chile central.

Figure 1. Minimum air temperature registered at 1,600, 2,800 and 3,600 m a.s.1. in the Andes of central Chile. 
hubo sólo 1 día con temperatura inferior a $0{ }^{\circ} \mathrm{C}$ y en $1.600 \mathrm{~m}$ no hubo eventos de temperaturas congelantes.

\section{CONDUCTANCIA HIDRÁUlica DE LA HOJA $(K f)$}

En general se encontró una disminución de la conductancia hidráulica foliar con la altitud. Los valores de $K f$ (Fig. 2) indican que éta es un 57\% mayor en plantas provenientes de los $1.600 \mathrm{~m}$, comparado con las provenientes de $2.800 \mathrm{~m}$ (Fisher test, $\mathrm{p}=0,03$ ) y un $63,8 \%$ (Fisher test, $\mathrm{p}<0,01$ ) superior al contrastar con las plantas provenientes de $3.600 \mathrm{~m}$. Al evaluar las diferencias entre las elevaciones mayores (2.800 y 3.600 $\mathrm{m})$, se encontró que $K f$ disminuyó en un $15,4 \%$ con la altitud (Fisher test, $\mathrm{p}<0,05$ ).

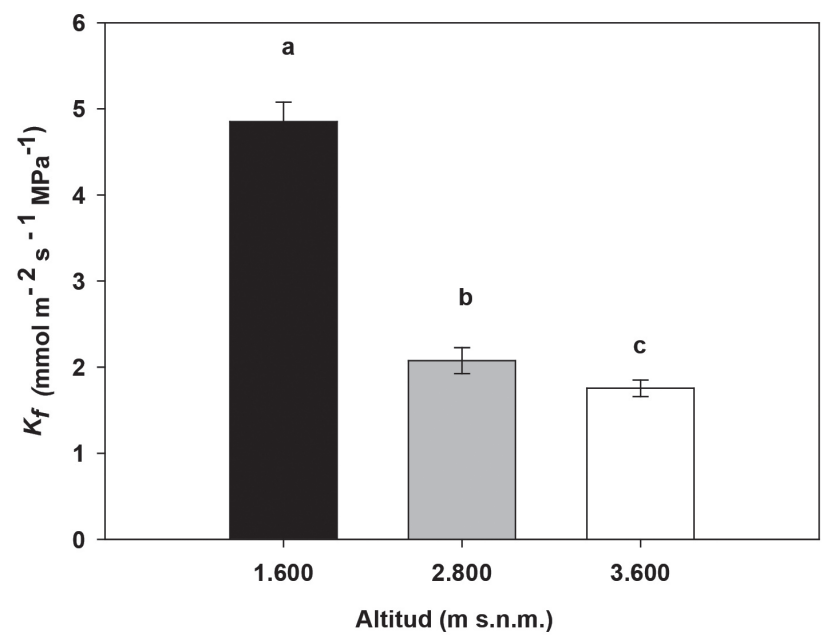

Figura 2. Conductancia hidráulica de la hoja $(K f)$ medida en terreno, en plantas de Phacelia secunda provenientes de 1.600, 2.800 y $3.600 \mathrm{~m}$ s.n.m. Los valores mostrados por las barras son el promedio de 5 réplicas \pm el error estándar de los datos y las letras indican las diferencias significativas entre altitud de acuerdo a Fisher test $(\mathrm{p}<$ $0,05)$.

Figure 2. Leaf hydraulic conductance $(K f)$ measured in Phacelia secunda plants at 1,600, 2,800 and 3,600 m a.s.l. Each bar correspond to mean $\pm \operatorname{SE}(n=5)$. Different letters denote statistically significant differences between elevations according to the Fisher test $(\mathrm{p}<0.05)$.

\section{DiÁMETRO Y NÚMERO DE VASOS XILEMÁTICOS}

El diámetro promedio de los vasos del xilema del pecíolo y de la vena central de las hojas presentó diferencias significativas con la altitud, siendo el diámetro de los vasos un $16 \%$ mayor en plantas provenientes de $1.600 \mathrm{~m}$, respecto a las otras dos altitudes (Fig. 3) (Fisher test, p $<0,01$ ). En las mediciones realizadas en el pecíolo de la hoja, las diferencias encontradas fueron mayores debido a que en este caso el diámetro de los vasos difiere entre un 32 y un $35 \%$ aproximadamente; siendo menor en plantas provenientes desde los $3.600 \mathrm{~m}$ (Fisher test, p < 0,01). Al estudiar la relación entre el diámetro de los vasos y la conductancia hidráulica de la hoja, se encontró una correlación positiva con un $\mathrm{R}^{2}$ de $0,746(\mathrm{p}<0,05)$. Por otro lado, se encontraron diferencias significativas entre el diámetro de los vasos de la lámina y el pecíolo de las hojas para una misma altitud (Fig. 3). Las plantas de $1.600 \mathrm{~m}$ presentaron vasos con un diámetro un $38,4 \%$ mayor en el pecíolo que en la lámina (Fisher test, p < 0,01). En las plantas de 2.800 y $3.600 \mathrm{~m}$ la diferencia entre la lámina y el pecíolo fue menor que las encontradas en las plantas de $1.600 \mathrm{~m}$, encontrándose mayor diámetro de vasos en el pecíolo que en la vena central de la lámina, la cual varió entre 17 y 12,8\% (Fisher test, p <0,05), respectivamente.

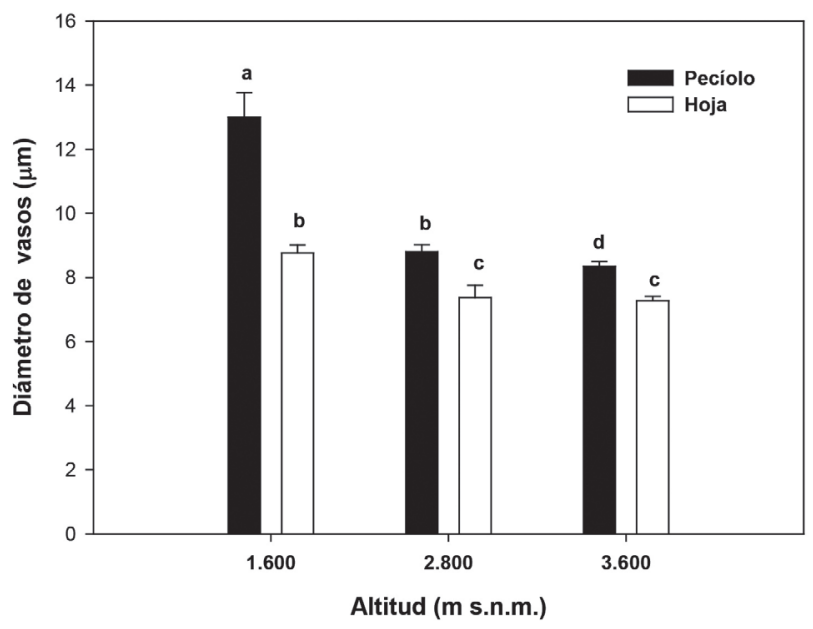

Figura 3. Diámetro de los vasos del tejido xilemático, en la lámina y pecíolo de la hoja, medido en cortes transversales de plantas de Phacelia secunda provenientes de $1.600,2.800$ y 3.600 m s.n.m. Los valores mostrados por las barras son el promedio de 5 replicas \pm el error estándar de los datos y las letras indican las diferencias significativas entre altitud y estructuras de la hoja (lámina y pecíolo), de acuerdo a Fisher test $(\mathrm{p}<0,05)$.

FIGURE 3. Diameter of the vessels of the xilematic tissue, in the lamina or blade and petiole of the leaf, measured in cross cuts of Phacelia secunda plants from 1,600, 2,800 and 3,600 m a.s.l. Each bar correspond to mean $\pm \mathrm{SE}(\mathrm{n}=5)$. Different letters denote statistically significant differences between elevations according to the Fisher test $(\mathrm{p}<0.05)$.

El número de vasos se correlacionó positivamente con la altitud (Fig. 4). Plantas de mayor altitud (2.800 y 3.600 $\mathrm{m})$ presentaron un mayor número de vasos xilemáticos comparado con las plantas provenientes de $1.600 \mathrm{~m}$ (Fisher test, $\mathrm{p}<0,001)$, tanto en la vena central de la lámina como en el pecíolo. Al comparar las altitudes extremas, dichas diferencias fueron cercanas al $21 \%$ entre el número de conductos del pecíolo y vena central de la lámina. En todas las altitudes se observó una disminución en el número de 
vasos desde el pecíolo hacia la vena central, siendo dicha diferencia en ca. de un $61 \%$ (Fisher test, $\mathrm{p}<0,001$ ).

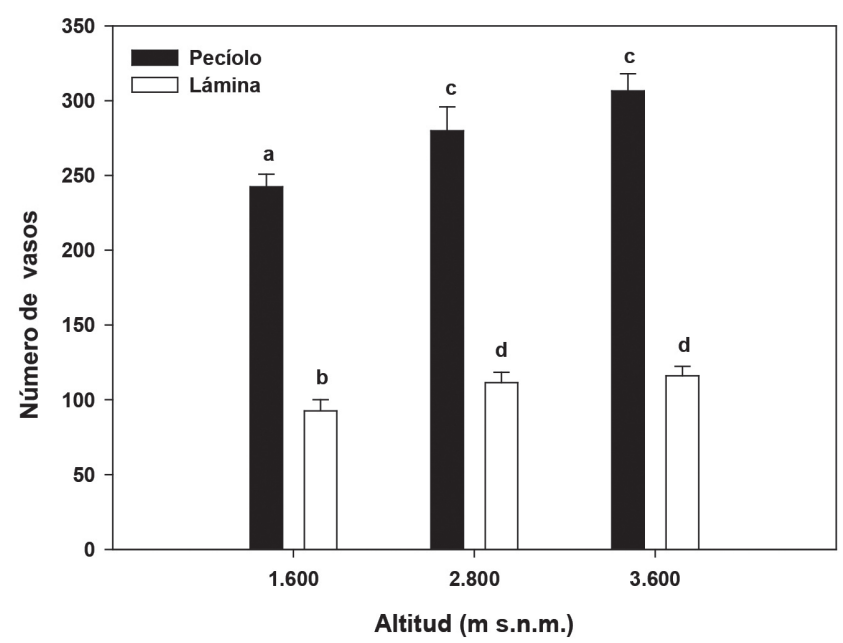

FiguRA 4. Número de vasos xilemáticos, en la lámina y pecíolo de la hoja, medido en cortes transversales de plantas de Phacelia secunda provenientes de $1.600,2.800$ y $3.600 \mathrm{~m}$ s.n.m. Los valores mostrados por las barras son el promedio de 5 replicas \pm el error estándar de los datos y las letras indican las diferencias significativas entre altitud y estructuras de la hoja (lámina y pecíolo), de acuerdo a Fisher test ( $\mathrm{p}$ $<0,05)$.

Figure 4. Vessels number, in the lamina or blade and petiole of the leaf, measured in cross cuts of Phacelia secunda plants from 1,600, 2,800 and 3,600 $\mathrm{m}$ a.s.l. Each bar correspond to mean $\pm \mathrm{SE}(\mathrm{n}=5)$. Different letters denote statistically significant differences between elevations according to the Fisher test $(\mathrm{p}<0.05)$.

ÍNDICE DE VULNERABILIDAD A LA CAVITACIÓN (IV) Y SU CORRELACIÓN CON EL DIÁMETRO DE VASOS

Los valores calculados para el índice de vulnerabilidad a la cavitación (IV), el cual indica la susceptibilidad del xilema a sufrir embolia, muestra que hay diferencias significativas con la altitud y entre lámina y pecíolo (Fig. 5). En la lámina, el IV disminuyó con la altitud de procedencia de las plantas. Las plantas de $1.600 \mathrm{~m}$ son entre un 37 y un $50 \%$ más vulnerables a la cavitación (Fisher test, $\mathrm{p}<0,01$ ) que las plantas de 2.800 y $3.600 \mathrm{~m}$. Estas diferencias se incrementan a un 60 y $71 \%$ en el pecíolo (Fisher test, $\mathrm{p}<0,01$ ). Si bien se encontraron diferencias entre las elevaciones mayores $(2.800$ y $3.600 \mathrm{~m})$, éstas fueron de menor magnitud. Las plantas de $3.600 \mathrm{~m}$ fueron entre un 23,5 y $37,7 \%$ menos vulnerables a la cavitación respecto a las plantas de 2.800, dependiendo del tejido que se compare (Fisher test, p < $0,05)$.

Al evaluar las diferencias de IV entre lámina y pecíolo, las mayores diferencias se encontraron para las plantas de $1.600 \mathrm{~m}$, en donde se encontró diferencias significativas de aproximadamente un 56\% mayor en el pecíolo que en la lámina (Fisher test, $\mathrm{p}<0,01$ ). Para las plantas de $2.800 \mathrm{y}$ $3.600 \mathrm{~m}$, esta diferencia fue menor y bordeó el 30\% (Fisher test, $\mathrm{p}<0,01)$.

En la relación entre el diámetro de los vasos y el índice de vulnerabilidad a la cavitación, se encontró una correlación positiva $\left(R^{2}=0,932 ; p<0.05\right)$, en donde a mayor diámetro de vasos hay una mayor probabilidad de cavitación.

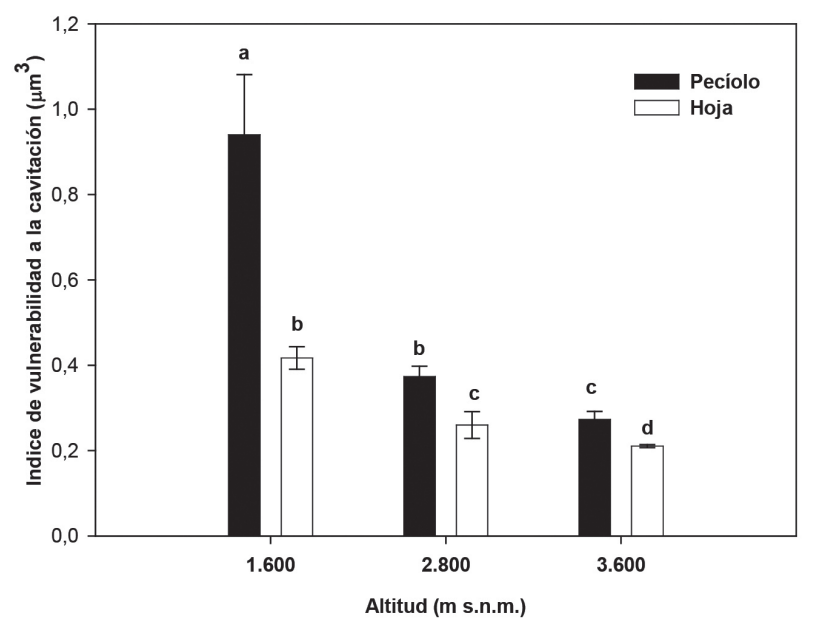

FigURA 5. Índice de vulnerabilidad a la cavitación (IV) para plantas de Phacelia secunda provenientes de $1.600,2.800$ y 3.600 m s.n.m para la lámina y pecíolo de la hoja. Los valores mostrados por las barras son el promedio de 5 replicas \pm el error estándar de los datos y las letras indican las diferencias significativas entre altitud y estructuras de la hoja (lámina y pecíolo), de acuerdo a Fisher test $(\mathrm{p}<0,05)$.

FigURE 5. Vulnerability to cavitation index (IV) for Phacelia secunda plants from 1,600, 2,800 and 3,600 m a.s.1, for the lamina or blade and petiole of the leaf. Each bar correspond to mean $\pm S E(n=5)$. Different letters denote statistically significant differences between elevations according to the Fisher test $(p<0.05)$.

\section{DISCUSIÓN}

El propósito de esta investigación fue determinar si plantas de $P$. secunda creciendo a diferentes altitudes contrastan en la capacidad hidráulica de su sistema conductor a nivel foliar y sus consecuencias sobre la vulnerabilidad a la cavitación. En particular se encontró que, en esta especie, la capacidad potencial para conducir agua a través de sus tejidos conductores disminuye con la altitud, a pesar del aumento en el número de vasos con la altitud. Esto último estaría relacionado con una disminución en la vulnerabilidad a la cavitación.

La respuesta de la conductancia hidráulica de la hoja, 
medida en plantas del gradiente altitudinal, permitió observar que en plantas provenientes desde $1.600 \mathrm{~m}$ la $K f$ fue mayor que en las plantas provenientes desde 2.800 y $3.600 \mathrm{~m}$. Esta pérdida en la conductancia hidráulica se correlaciona con la disminución observada en el diámetro de los vasos (Fig. 3), lo cual es coherente ya que gran parte de la resistencia de una hoja al flujo de agua es su vascularización (Sack et al. 2004).

En los resultados se encontró que el tamaño de los conductos en Phacelia secunda estarían principalmente determinados por la temperatura, ya que fueron las plantas de mayor altitud $(3.600 \mathrm{~m})$ las que presentaron un menor diámetro de conductos xilemáticos acompañado esto de una menor vulnerabilidad a la cavitación. Sin embargo, la capacidad hidráulica no sólo depende del tamaño de los vasos, sino también del número de vasos por nivel (McCulloh et al. 2003, 2004, Atala \& Lusk 2008). Los resultados muestran un incremento en el número de vasos en las plantas provenientes de mayor altitud. Sin embargo, este incremento en el número de vasos a mayor altitud no fue suficiente para compensar la mayor resistencia impuesta por la reducción en el tamaño de los vasos.

Diferencias en el diámetro de los vasos entre plantas podrían estar relacionadas a diferencias ontogenéticas y/o alométricas (Iqbal \& Gouse 1977, West et al. 1999, Weitz et al. 2006). Desde la perspectiva ontogenética, plantas juveniles tienden a tener diámetros de vasos menores que plantas adultas (Carlquist 1975, Iqbal \& Gouse 1977). En el presente caso es poco probable que diferencias ontogenéticas estén detrás de lo aquí reportado ya que se trabajó sólo con plantas adultas de $P$. secunda. Por otro lado, es conocido que en plantas de alta-montaña, para una misma especie, los individuos de poblaciones ubicadas a mayores elevaciones son más longevos que los de altitudes menores (Körner 2003, Von Arx et al. 2006). Desde una perspectiva alométrica, plantas más grandes tendrían vasos más grandes que plantas pequeñas, pudiendo modificar en forma indirecta la capacidad de transporte en las plantas (West et al. 1999, Enquist 2003). En nuestro caso esta explicación también parece poco probable ya que los individuos de $P$. secunda que habitan las mayores elevaciones forman rosetas de mayor tamaño que los de altitudes menores (Cavieres 2000).

Tal como ocurre en muchas montañas del mundo, los eventos de temperaturas congelantes aumentan a mayores elevaciones. Estos eventos de congelamiento pueden producir embolismo por la formación de burbujas de gas, las cuales se pueden expandir con el descongelamiento, pudiendo provocar la cavitación (Mayr \& Cochard 2003). Por esta razón, las plantas frecuentemente expuestas a estos eventos tienden a presentar un diámetro menor de los vasos, disminuyendo así la vulnerabilidad a la cavitación (Davis et al. 1999). Los resultados obtenidos corroboran todo lo anterior.

West et al. (1999), asume una disminución acropétalo en el área conductora con un número constante de vasos y un ahusamiento progresivo de los vasos desde la base al ápice.
En cambio, McCulloh et al. (2003) propone un incremento en el número de vasos y el área conductora desde la base hacia el ápice. En este trabajo se observó una disminución en el número de vasos desde el pecíolo a la vena central de la hoja, acompañado de un mayor ahusamiento de éstos en la vena central de la hoja. Este ahusamiento de los vasos podría amortiguar el incremento en la resistencia, producto de la reducción de los vasos (Becker et al. 2000), ya que vasos más estrechos podrían actuar como un sistema de respaldo cuando se produce embolismo (Jiménez-Castillo \& Lusk 2013). En consecuencia, en $P$. secunda habría un apoyo a lo sugerido por West et al. (1999), disminuyendo el área conductora y mostrando un evidente ahusamiento en los vasos. Sin embargo, en $P$. secunda el número de vasos no es constante.

Al comparar los diámetros de vasos y la vulnerabilidad al embolismo (IV) entre la vena central de la hoja y pecíolo a las diferentes altitudes, se encontró un mayor contraste entre estos dos tejidos en plantas de $1.600 \mathrm{~m}$. En estas plantas el pecíolo tiene un diámetro de vasos mayor que el de la vena central, lo cual determina una mayor vulnerabilidad a la cavitación. Es sabido que los vasos de la vena central provienen del pecíolo (Sack \& Frole 2006); sin embargo, ésta se ramifica en venas laterales de distinto orden, por lo cual es probable que la conductividad hidráulica del pecíolo sea proporcionalmente mayor en hojas con una mayor área foliar. Adicionalmente, los pecíolos de las hojas de $1.600 \mathrm{~m}$ son aproximadamente un $47 \%$ más largos que los de las otras dos elevaciones y por tanto ofrecerán más resistencia a un mismo diámetro de vaso. Estas diferencias morfológicas con la elevación también han sido reportadas en estudios anteriores en $P$. secunda, en donde se encontró una tendencia a privilegiar el crecimiento vertical a bajas altitudes y el crecimiento horizontal en altitudes mayores, la cual sería una respuesta adaptativa a las condiciones frías de los ambientes de alta montaña (Cavieres 2000). De acuerdo a Cavieres \& Arroyo (1999), la diferenciación morfológica y reproductiva (e.g. proceso de germinación y formación de bancos de semillas) observada en este gradiente altitudinal correspondería a diferenciación ecotípica, sustentada por la baja probabilidad de intercambio genético entre poblaciones ubicadas en altitudes diferentes debido a los desfases en la floración y los cambios en los gremios de polinizadores (Arroyo et al. 1981, 1982). Sin embargo, que exista esta diferenciación ecotípica a nivel morfológico no implica que ocurra lo mismo con caracteres anatómicos, como por ejemplo las diferencias encontradas en los vasos xilemáticos con la altitud. En consecuencia no descartamos que para este rasgo en particular exista plasticidad fenotípica y estudios más detallados son necesarios para abordar este tema.

Debido a que la mayor limitante para fotosintetizar a mayor altitud seria la baja temperatura (Sanfuentes et al. 2012), se esperaría una respuesta altamente favorable de la fotosíntesis al incremento de la temperatura. Sin embargo, la menor $K f$ también se correlaciona con menores tasas de 
conductancia estomática y de asimilación de carbono, por lo cual las plantas que ven disminuida su $K f$ ven reducida directamente sus tasas fotosintéticas (Nardini et al. 2008, Hubbard et al. 2001, Resco et al. 2008, Brodribb \& Field 2010). Esto podría llegar a ser una limitante en la respuesta fotosintética de estas plantas al incremento en la temperatura proyectada por el cambio climático. Además, los diferentes escenarios de cambio climático propuestos para nuestra zona de estudio, predicen disminuciones en las precipitaciones durante la época estival (CONAMA 2006, IPCC 2007), lo que podría incrementar el déficit hídrico y generar escenarios adversos para plantas que presentan una mayor vulnerabilidad a la cavitación debido a su morfología interna.

Durante este estudio no se evaluó el efecto que pueden tener las restricciones hídricas en la anatomía y capacidad de transportar agua en $P$. secunda. Los distintos parámetros evaluados fueron determinados al comienzo de la estación de crecimiento de las plantas, época en la cual no hay diferencias significativas en la disponibilidad de agua con la elevación (Cavieres et al. 2006). En consecuencia, al menos en la fenología estudiada no se observó alteraciones en la capacidad hidráulica de las plantas producto del déficit hídrico. Probablemente, esto se deba a que las plantas generan la mayor parte de su follaje bajo suficiencia hídrica (inicio y mediados de la estación de crecimiento). De aquí se desprende que la estrategia de las poblaciones más bajas donde hay sequía estival al final de la estación de crecimiento sería maximizar la fotosíntesis, el crecimiento y la expansión foliar muy temprano en la estación de crecimiento, favoreciendo que esto ocurra con un suministro apropiado de agua en el suelo. Esto aseguraría las reservas para el inicio de la siguiente temporada y el gasto energético reproductivo. Sería interesante contrastar estos resultados con hojas expandidas bajo condiciones limitantes de agua en las tres procedencias. Por esto, las respuestas y mecanismos que desarrollen estas plantas para tolerar o evadir estos factores de estrés ambiental son relevantes tanto para la supervivencia como para la distribución de esta especie en la Cordillera de los Andes de Chile Central.

En conclusión, los resultados obtenidos en este estudio indican que en plantas de $P$. secunda existe una correlación negativa entre la conductancia hidráulica de la hoja con la altitud. Esto podría ser una respuesta a la disminución de la temperatura promedio con la altitud, y del incremento en los eventos de temperaturas congelantes durante la temporada de crecimiento de las plantas. Esto trae como consecuencia una disminución del tamaño de los conductos xilemáticos, viéndose disminuida la $K f$. Además, se encontró una correlación negativa entre la vulnerabilidad a la cavitación con las altitudes en estudio, encontrándose que las plantas de menores altitudes, en donde el diámetro de los vasos xilemáticos es mayor, hay más probabilidad de cavitación. Por lo tanto, este atributo anatómico-funcional estaría prioritariamente determinado por las temperaturas de crecimiento de las diferentes altitudes.

\section{AGRADECIMIENTOS}

Este estudio fue financiado por FONDECYT 1090389 y por los Proyectos CONICYT PFB-023 y P05-02 de la Iniciativa Científica Milenio del Ministerio de Economía, Fomento y Turismo del Instituto de Ecología y Biodiversidad IEB. C. Hernández-Fuentes agradece a la Beca Doctoral CONICYT.

\section{BIBLIOGRAFÍA}

Arroyo, M.T.K, J.J. Armesto \& C. Villagrán. 1981. Plant phenological patterns in the high Andean Cordillera of Central Chile. Journal of Ecology 69: 205-223.

Arroyo, M.T.K., R. Primack \& J. Armesto. 1982. Community studies in pollination ecology in the high temperate Andes of Central Chile. I. Pollination mechanisms and altitudinal variation. American Journal of Botany 69(1): 82-97.

Atala, C. \& C.H. Lusk. 2008. Xylem anatomy of Betula pendula roth samplings: relationship to physical vascular models. Gayana Botánica 65(1): 18-27.

Becker, P., R.J Gribben \& C.M. Lim. 2000. Tapered conduits can buffer hydraulic conductance from path-length effects. Tree Physiology 20: 965-967.

Bовнм, J. 1893. Capillarität und Saftsteigen. Berichte der Deutschen Botanischen Gesellschaft 11(3): 203-212.

BOYer, J.S. 1976. Photosynthesis at low water potentials. Philosophical Transactions of the Royal Society B 273(927): 501-512.

BrodribB, T.J., T.S. Field \& G.J. Jordan. 2007. Leaf maximum photosynthetic rate and venation are linked by hydraulics. Plant Physiology 144(4): 1890-1898.

BRodribB, T.J. \& T.S. FIELD. 2010. Leaf hydraulic evolution led a surge in leaf photosynthetic capacity during early angiosperm diversification. Ecology Letters 13(2): 175-183.

Carlquist, S. 1975. Ecological Strategies of Xylem Evolution. University of California Press, Berkeley, CA, USA. 259 pp.

CAVIERES, L.A. 2000. Variación morfológica de Phacelia secunda J.F.Gmel. (Hydrophyllaceae) a lo largo de un gradiente altitudinal en Chile Central. Gayana Botánica 57(1): 89-96.

Cavieres, L.A. \& M.T.K. Arroyo. 1999. Tasa de enfriamiento adiabático del aire en el valle del río Molina, Provincia de Santiago, Chile central $\left(33^{\circ} \mathrm{S}\right)$. Revista Geográfica de Chile Terra Australis 44: 79-86.

Cavieres, L.A., E. Badano, A. Sierra-Almeida, S. GonzÁlez-Gómez \& M. Molina-Montenegro. 2006. Positive interactions between alpine plant species and the nurse cushion plant Laretia acaulis do not increase with elevation in the Andes of central Chile. New Phytologist 169(1): 59-69.

Cavieres, L.A., E. Badano, A. Sierra-Almeida \& M. MolinaMontenegro. 2007. Microclimatic modifications of cushion plants and their consequences for seedling survival of native and non-native herbaceous species in the High Andes of Central Chile. Arctic, Antarctic and Alpine Research 39(2): 229-236. 
Gayana Bot. 72(1), 2015

Choat, B., D.E. Medek, S.A. Stuart, J. Pasquet-Kok, J.J.G. Egerton, H. Salari, L. Sack \& M.C. Ball. 2011. Xylem traits mediate a trade-off between resistance to freezethaw-induced embolism and photosynthetic capacity in overwintering evergreens. New Phytologist 191(4): 9961005.

CONAMA. 2006. Estudio de la variabilidad climática en Chile para el siglo XXI. Informe Final. Departamento de Geofísica, Facultad de Ciencias Físicas y Matemáticas, Universidad de Chile. Santiago, Chile 63 pp.

Davis, S., J. Sperry \& U. Hacke. 1999. The relationship between xylem conduit diameter and cavitation caused by freezing. Annual Botanical 86(10): 1367-1372.

Dixon, H. \& J. JoLy. 1894. On the ascent of sap. Philosophical Transactions of the Royal Society of London B 186: 563-576.

ENQuist, B. 2003. Cope's rule and the evolution of long-distance transport in vascular plants: allometric scaling, biomass partitioning and optimization. Plant, Cell \& Environment 26(1): 151-161.

EWERs, F. \& J. FISCHER. 1989. Variation in vessel length and diameter in stems of six tropical and subtropical lianas. American Journal of Botany 76(10): 1452-1459.

Hacke, U. \& J. Sperry. 2001. Functional and ecological xylem anatomy. Perspectives in Plant Ecological Evolution System 4: 97-115.

Hubbard, R.M., V. Stiller, M.G. Ryan \& J.S. Sperry. 2001. Stomatal conductance and photosynthesis vary linearly with plant hydraulic conductance in ponderosa pine. Plant, Cell \& Environment 24(1): 113-121.

iPCC (Intergovernmental Panel On Climate Change). 2007. Climate Change Impacts, Adaptation and Vulnerability. Cambridge University Press. 103 pp.

Iqbal, M. \& A. Gouse. 1977. Ontogenetic size variation of sievetube elements in Prosopis spicigera L. Bulletin de la Societe Botanique de France 124: 445- 450.

JACKSON, G.E. \& J. Grace. 1994. Cavitation and water transport in trees. Endeavour 18: 50-54.

JimÉneZ-CASTillo, M. \& C.H. LuSK. 2013. Comparative vascular anatomy and function of woody plants in a temperate rainforest: lianas suffer higher levels of freeze-thaw embolism than associated trees. Functional Ecology 27: 403-412.

KöRnER, C. 2003. Alpine Plant Life ( $2^{\text {nd }}$ ed.). Springe Verlag, Berlin, Germany. 349 pp.

Martre, P., H. Cochard \& J.L. Durand. 2001. Hydraulic architecture and water flow in growing grass tillers (Festuca arundinacea Schreb.). Plant Cell \& Environment 24(1): 65-76.

MaYr, S. \& H. COCHARD. 2003. A new method for vulnerability analysis of small xylem areas reveals that compression wood of Norway spruce has lower hydraulic safety than opposite wood. Plant, Cell \& Environment 26(8): 1365-1371.

Mayr, S., P. Schmid, J. Laur, S. Rosner, K. Charra-Vaskou, B. DÄMON \& U.G HACKE. 2014. Uptake of water via branches helps timberline conifers refill embolized xylem in late winter. Plant Physiology 164: 1731-1740.

McCulloh, K.A., J.S. Sperry \& F.R. Adler. 2003. Water transport in plants obeys Murray's law. Nature 421: 939-942.

McCulloh, K.A., J.S. Sperry \& F.R. Adler. 2004. Murray's law and the hydraulic vs mechanical functioning of wood. Functional Ecology 18: 931-938.

Mencuccini, M. 2003. The ecological significance of long-distance water transport: short-term regulation, long-term acclimation and the hydraulic costs of stature across plant life forms. Plant, Cell \& Environment 26(1): 163-182.

NARDINI, A. \& S. SALLEO. 2000. Limitation of stomatal conductance by hydraulic traits: sensing or preventing xylem cavitation? Trees 15: 14-24.

Nardini, A., M.T. Tyree \& S. Salleo. 2001. Xylem cavitation in the leaf of Prunus laurocerasus and its impact on leaf hydraulics. Plant Physiology 125: 1700-1709.

Nardini, A., E. Gortan, M. Ramani \& S. Salleo. 2008. Heterogeneity of gas exchange rates over the leaf surface in tobacco: an effect of hydraulic architecture? Plant, Cell \& Environment 31(6): 804-812.

Poorter, L., I. McdDonald, A. Alarcón, E. Fichtler, J.C. Licona, M. Peña-Claros, F. Sterck, Z. Villeggas \& U. SAss-KlaAssen. 2010. The importance of wood traits and hydraulic conductance for the performance and life history strategies of 42 rainforest tree species. New Phytologist 185(2): 481-492.

Price, C.A., B.J. Enquist \& V.M. Savage. 2007. A general model for allometric covariation in botanical form and function. Proceedings of the National Academy of Sciences, USA 104: 13204-13209.

Resco, V., D.D. Ignace, W. Sun, T.E. Huxman, J.F. Weltzin \& D.G. WiLLIAMs. 2008. Chlorophyll fluorescence, predawn water potential and photosynthesis in precipitation pulse-driven ecosystems - implications for ecological studies. Functional Ecology 22(3): 479-483.

Reyes-Santamaría, I., A. Barrientos-Priego, T. Terrazas \& C. Trejo. 2002. Xylem conductivity and vulnerability in cultivars and races of avocado. Scientia Horticulturae 92: 97-105.

Richter, H. 1972. Wie entstehen Saugspannungs gradienten in Bäumen? Berichte der Deutschen Botanischen Gesellschaft 85: 341-351.

Russo, S.E., K.L. Jenkins, S.K. Wiser, M. URiarte, R.P. Duncan \& D.A. CoOmes. 2010. Interspecific relationships among growth, mortality and xylem traits of woody species from New Zealand. Functional Ecology 24(2): 253-262.

Sack, L., P.D. Cowan \& N.M. Holbrook. 2003. The major veins of mesomorphic leaves revisited: testing for conductive overload in Acer saccharum (Aceraceae) and Quercus rubra (Fagaceae). American Journal of Botany 90(1): 32-39.

SACK, L. \& N.M. Holbrook. 2006. Leaf hydraulics. Annual Review of Plant Biology 57: 361-381.

SACK, L. \& K. Frole. 2006. Leaf structural diversity is related to hydraulic capacity in tropical rainforest trees. Ecology 87(2): 483-491.

SAck, L., P. Melcher, M. Zwieniecki \& N. Holbrook. 2002. The hydraulic conductance of the angiosperm leaf lamina: a comparison of three measurement methods. Journal of Experimental Botany 53(378): 2177-2184.

SAck, L., C. Streeter \& N. Holbrook. 2004. Hydraulic analysis of water flow through leaves of sugarmaple and red oak. Plant Physiology 134: 1824-1833.

SACK, L. \& M.T. TyreE. 2005. Leaf hydraulics and its implications in plant structure and function. In: N.M. Holbrook \& M.A. Zwieniecki (eds.), Vascular Transport in Plants. Elsevier/ Academic Press, Oxford 93-114 pp.

Sanfuentes, C., A. Sierra-Almeida \& L.A. Cavieres. 2012. Efecto del aumento de la temperatura en el desempeño fotosintético de una especie alto-andina en dos altitudes contrastantes. Gayana Botánica 69(1): 37-45. 
Conductancia hidráulica foliar en un gradiente altitudinal: HeRnÁndEZ-FuENTES, C. ET AL.

Santibáñez, F. \& J.M. Uribe. 1990. Atlas agroclimático de Chile: Región V y Metropolitana. Universidad de Chile, Facultad de Ciencias Agrarias y Forestales, Santiago. 99 pp.

Sierra-Almeida, A., L.A. Cavieres \& L.A. Bravo. 2009. Freezing resistance varies within the growing season and with elevation in high-Andean species of central Chile. New Phytologist 182(2): 461-468.

Tyree, M. \& M. Zimmermann. 2002. Xylem structure and the ascent of sap. Springer, Berlin, Germany. 285 pp.

TYREE, M.T. 2003. Hydraulic limits on tree performance: Transpiration, carbon gain and growth of trees. Trees 17(2): 95-100.
Von Arx, G., P.J. Edwards \& D. Hansjörg. 2006. Evidence for life history changes in high-altitude populations of three perennial forbs. Ecology 87(3): 665-674.

Weitz, J.S., K. Ogle \& H.S. Horn. 2006. Ontogenetically stable hydraulic design in woody plants. Functional Ecology 20: 191-199.

West, G.B., J.H. Brown \& B.J. EnQuist. 1999. A general model for the structure and allometry of plant vascular systems. Nature 400: 664-667.

Zimmermann, M.H. 1983. Xylem structure and the ascent of sap. Springer-Verlag, Berlin, Germany. 285 pp.

Recibido: 22.05 .14

Aceptado: 17.02.15 